doi:10.17659/01.2020.0063

Journal of Case Reports 2020;10(4):237-240

\title{
Guiding Catheter-Induced Dissection of the Left Main Coronary Artery: Percutaneous Coronary Intervention or Surgery?
}

\author{
Santosh Kumar Sinha, Vinay Krishna \\ Department of Cardiology, LPS Institute of Cardiology, Ganesh Shankar Vidyarthi Memorial Medical College, Kanpur, \\ Uttar Pradesh, India.
}

\section{Corresponding Author:}

Dr Santosh Kumar Sinha

Email: fionasan@rediffmail.com

This is an Open Access article distributed under the terms of the Creative Commons Attribution License (creativecommons.org/ licenses/by/3.0).

Received : May 10,2020

Accepted : August 21, 2020

Published : November 20, 2020

\begin{abstract}
Background: Catheter-induced left main coronary artery (LMCA) dissection, though quite rare, is one of the most dreaded and life-threatening complication of coronary intervention and requires urgent management because if not promptly diagnosed and treated with myocardial revascularization, it can have fatal consequences. Case Report: Here, we report a case iatrogenic dissection of left main coronary artery by vigorous manipulation of extra backup (EBU) guiding catheter in a 73-year old male during revascularization of proximal left anterior descending artery which was successfully bailed out by stenting of left main artery using $4 \times 23 \mathrm{~mm}$ Xience Prime everolimus eluting stent (Abott Vascular; USA) at $12 \mathrm{~atm}$ pressure achieving complete sealing of flap with TIMI III flow. Conclusion: Coronary artery dissection can be precipitated by catheter manipulation.
\end{abstract}

Keywords: Coronary Vessels, Dissecting Aneurysm, Iatrogenic Disease, Myocardial Revascularization, Stents.

\section{Introduction}

Left main coronary artery (LMCA) either primary or secondary is harbinger to catastrophic vessel closure. It can be precipitated by manipulation of hardwares in LM ostium or during intervention of the ostial lesion of the left anterior descending artery (LAD). The incidence of dissection occurring during catheter-based procedures is around $0.1 \%[1,2]$. Secondary coronary artery dissections may be due to an anterograde extension of an aortic dissection or iatrogenic during manipulation during routine catheterization or intervention. It can complicate into embolization, ischaemia, perforation, and terminally into ventricular fibrillation [3].

\section{Case Report}

A 68-year-old diabetic and smoker male presented with exertional angina-Canadian Cardiovascular
Society (CCS) - class II for past 4 years. His hemogram and routine biochemistry were normal. An electrocardiogram showed mild ST depression in V1-4 with preserved R wave. Cardiac markers for necrosis were negative. Echocardiography revealed mild concentric left ventricular hypertrophy, grade-II diastolic dysfunction, and normal systolic function with an ejection fraction of $60 \%$.

Coronary angiogram was performed after proper consent through transfemoral route which revealed critical lesion in proximal LAD with $90 \%$ stenosis [Fig.1]. Ascending aorta was dilated as LMCA could not be cannulated with Judkins left (JL) 3.5 diagnostic catheter. It was cannulated with JL 4 catheter with difficulty. LMCA could not be hooked with 6F extra backup guide catheter 3.5 (EBU- Medtronic, USA). Somehow it was cannulated with bigger curve (EBU 4). Lesion was 
crossed with 0.014" run through wire (Terumo, Japan) and was gradually predilated using $2 \times 10$, and $2.5 \times 10 \mathrm{~mm}$ sapphire semicompliant balloons (Orbus Neisch, Netherland). $3 \times 18 \mathrm{~mm}$ Endeavour Resolute Zotarolimus eluting stent (Medtronic, USA) was tracked across the lesion but could not be properly placed as guiding was not properly cannulating the ostium of left main artery [Fig.2a,b]. EBU was little withdrawn and pushed over the wire which led to deep throttling of left main artery [Fig.3a]. Stent was deployed at 12 atm pressure where the monitor showed ventricularization of pressure trace. Guiding catheter was little withdrawn and contrast injection showed dissection of LMCA as patient had severe chest pain, profound hypotension as blood pressure fell down to $60 / 40 \mathrm{mmHg}$ and ST elevation in V1-6 [Fig.3b,4a]. Intravenous infusion of normal saline was started. In order to salvage the patient, LMCA was stented quickly with $4 \times 18 \mathrm{~mm}$ Endeavour Resolute stent at 14 atm pressure which stabilized the blood pressure and ST changes [Fig.4b]. As there was another segment of LAD which was uncovered with stent, another $3.5 \times 12 \mathrm{~mm}$ Endeavour Resolute stent was deployed between the two previous stent at $14 \mathrm{~atm}$ pressure [Fig.4c]. All the deployed stents were post-dilated using $4 \times 10 \mathrm{~mm}$ and $3.5 \times 10 \mathrm{~mm}$ non-compliant balloon at high pressure. Post-procedure, blood pressure rose to $112 / 48 \mathrm{mmHg}$ with TIMI III flow and normalization of ST changes [Fig.4d]. He was discharged in stable condition with ticagrelor 180 $\mathrm{mg}$, aspirin $75 \mathrm{mg}$, rosuvastatin $40 \mathrm{mg}$, metoprolol $100 \mathrm{mg}$, ramipril $10 \mathrm{mg}$, and gilbenclaimide $2 \mathrm{mg}$ once daily. He is in regular follow up since then.

\section{Discussion}

Catheter-induced left main coronary artery dissection is a rare but well-recognized lifethreatening complication of coronary intervention. Emergent coronary artery bypass surgery (CABS) is indicated and has been associated with good clinical outcomes. In our case, it was either distal dissection of left main or proximal dissection

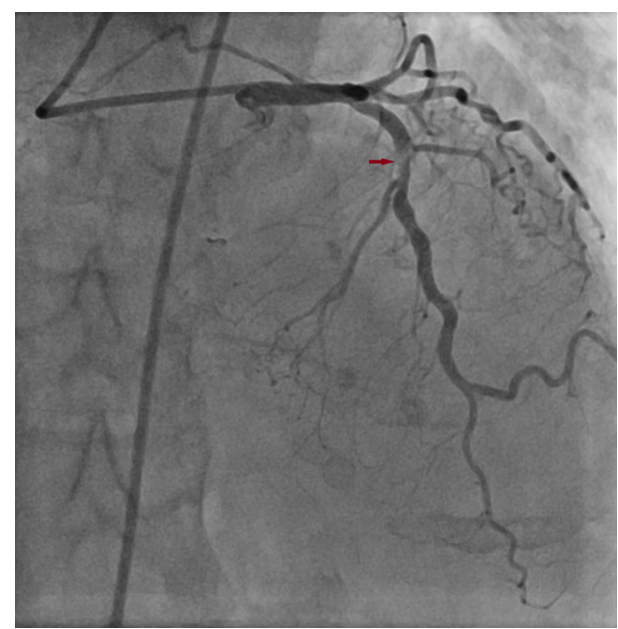

Fig.1: Coronary angiogram revealing critical lesion with haziness (red arrow) in proximal LAD with $90 \%$ stenosis.

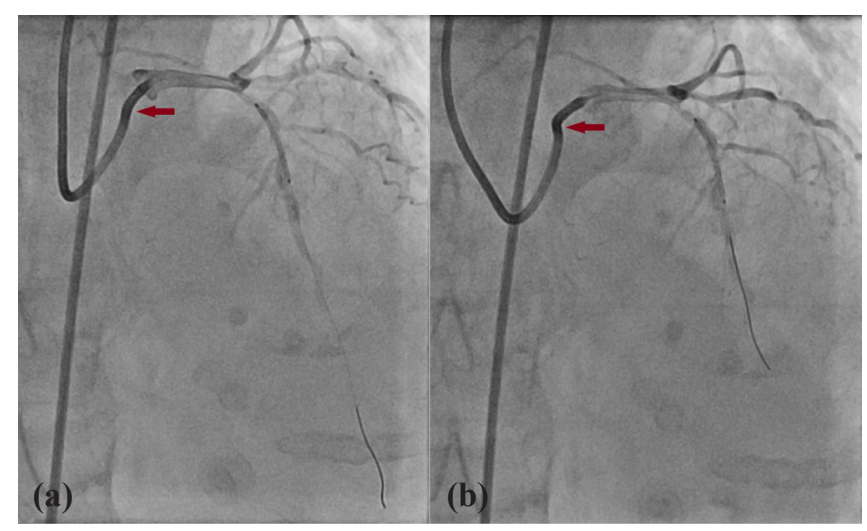

Fig.2: LMCA was cannulated with bigger curve (EBU 4) but had non-coaxial alignment (red arrow) which was causing difficulty in placing stent across the lesion.

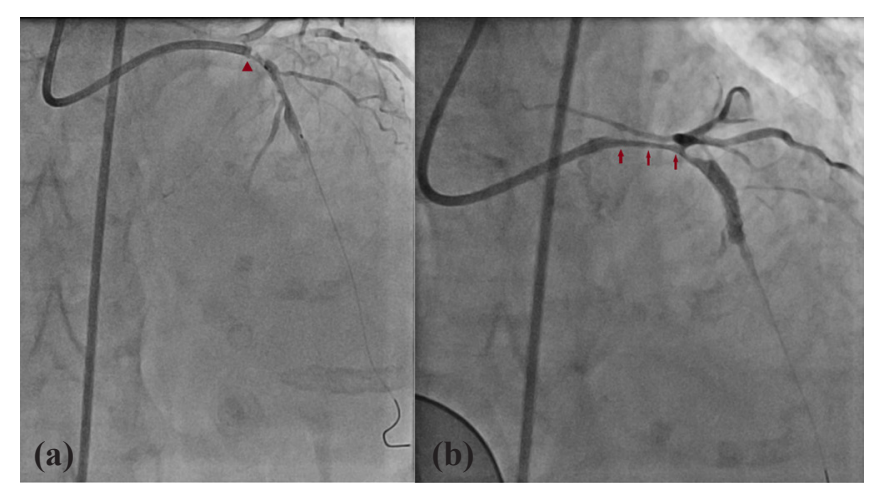

Fig.3:(a) EBU was little withdrawn and pushed over the wire which led to deep throttling of left main (red arrowhead); (b) guiding catheter was little withdrawn and contrast injection showed dissection of LMCA (red arrows). 
of ostium of LAD which progressed retrograde involving the whole of left main artery. Although the exact mechanism of iatrogenic left main dissection with retrograde progression is not exactly known, mechanism is progressive retrograde dispersion of the sub-intimal space created by entry point which originates within the dissection plane as a result of trauma induced by guide catheter. These can be result of vigorous hand injection of contrast medium, sub-intimal passage of the guidewire, and/or inadvertent handling of the guiding catheter. Predisposing factors are cystic medial necrosis, hypertension, Marfan syndrome, and atherosclerosis. Plaque disruption may serve as an entry point for the pulsatile flow of blood as atherosclerosis also weakens the media.

Iatrogenic LMCA dissection results from excessive manipulation of the catheter; changing catheters to others with greater curvature or of different forms (Amplatz), to overcome difficulties found in LMCA catheterization due to its unusual location at the aortic root; vigorous contrast medium injection with the catheter in a no coaxial alignment; use of hard-tip catheters; and deep intubation of the catheter [1,2]. In our case, vigorous manipulation as well as deep engagement, both were responsible. EBU guide (Medtronic; USA) are relatively stiffer compared to XBU (Cordis Corp; USA) or Mac 1(Boston Scientific; USA). Management of iatrogenic left main dissection stent implantation is an appropriate alternative to coronary artery bypass graft surgery, because it is a rapid and effective treatment [2]. Conservative treatment is applicable for hemodynamically stable patient with a low-grade dissection [2]. Intervention is indicated for hemodynamic instability. Catheter based intervention, in the form of stenting (covered stent, bare metal stent, drug eluting stent) of the coronary dissection entry point, is reserved for patients with "limited" aortic involvement (within $40 \mathrm{~mm}$ from the coronary ostium) [2]. IABP and temporary pacing may be needed in some cases but sudden and rapid downhill hardly gives any time,

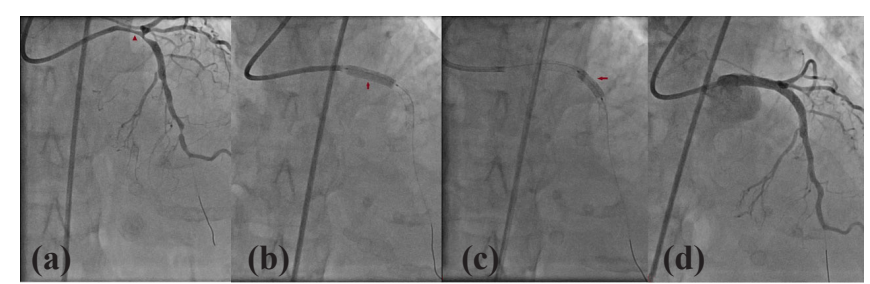

Fig.4:(a,b) LMCA was stented quickly with $4 \times 18 \mathrm{~mm}$ Endeavour Resolute stent therefore sealing the flap; (c) segment of LAD which was uncovered with stent, another $3.5 \times 12 \mathrm{~mm}$ Endeavour Resolute stent was deployed between the two previous stent at 14 atm pressure; (d) Post procedure showing TIMI III flow.

and therefore timely recognition of the isolated left main dissection is of paramount importance as early and prompt stenting of the entry point in the coronary tree may prevent retrograde aortic extension. Survival rates of approximately 90\% and restenosis rates at six months ranging from $0-30 \%$ have been demonstrated in small series of patients with iatrogenic LMCA dissection over 1230 months period. One should remain vigilant and perform angiography to rule out LMCA restenosis as they may manifest as sudden death $[5,6]$.

\section{Conclusion}

Catheter-induced left main coronary artery dissection can be precipitated by vigorous manipulation. Early recognition of the isolated left main dissection and prompt stenting of the entry point in the coronary tree may prevent further complication.

Contributors: SKS: Drafting the work and patient management; VK: substantial contributions to the conception and critical review of the work. SKS will act as a study guarantor. Both authors approved the final version of the manuscript and are responsible for all aspects of the study.

Funding: None; Competing interests: None stated.

\section{References}

1. Ertas G, Ural E, Van Der Giessen WJ. Successful stenting of catheter-induced unprotected left main coronary artery dissection. Cardiovasc J Afr. 2012;23:e6-e7.

2. Awadalla H, Sabet S, Sebale A, Rosales O, Smalling R. Catheter-induced left main dissection incidence, predisposition and therapeutic strategies experience from two sides of the hemisphere. J Invasive Cardiol. 2005; 17:233-236. 
3. Boyle AJ, Chan M, Dib J, Resar J. Catheter-induced coronary artery dissection: Risk factors, prevention and management. J Invasive Cardiol. 2006;18:500-503.

4. Awadalla H, Salloum JG, Smalling RW, Sdringola S. Catheter-induced dissection of the left main coronary artery with and without extension to the aortic root: A report of two cases and a review of the literature. J Interven Cardiol. 2004;17:253-257.
5. Onsea K, Kayaert P, Desmet W, Dubois CL. Iatrogenic left main coronary artery dissection. Neth Heart J. 2011;19:192-195.

6. Lee SW, Hong MK, Kim YH, Park JH, Rhee K, Lee $\mathrm{CW}$, et al. Bail-out stenting for left main coronary artery dissection during catheter-based procedure: acute and long-term results. Clin Cardiol. 2004;27:393-395. 